\title{
Orthogonal 3D Shapes of Theta Graphs ${ }^{\star}$
}

\author{
Emilio Di Giacomo ${ }^{1}$, Giuseppe Liotta ${ }^{1}$, and Maurizio Patrignani ${ }^{2}$ \\ 1 Università di Perugia, Italy \\ \{digiacomo, liotta\}@diei.unipg.it \\ ${ }^{2}$ Università di Roma Tre, Italy \\ patrigna@dia.uniroma3.it
}

\begin{abstract}
The recent interest in three dimensional graph drawing has been motivating studies on how to extend two dimensional techniques to higher dimensions. A common approach for computing a $2 \mathrm{D}$ orthogonal drawing of a graph separates the task of defining the shape of the drawing from the task of computing its coordinates. First results towards finding a three-dimensional counterpart of this approach are presented in [89], where characterizations of orthogonal representations of paths and cycles are studied. In this note we show that the known characterization for cycles does not immediately extend to even seemingly simple graphs such as theta graphs. A sufficient condition for recognizing three-dimensional orthogonal representations of theta graphs is also presented.
\end{abstract}

\section{Introduction}

The recent interest in three-dimensional graph drawing has been motivating studies on how to extend two dimensional techniques to 3D space. Work in this direction includes extensions of simulated annealing techniques, spring embedder techniques, and incremental techniques (see e.g., [3/5/11/15]). However, while a rich body of literature is devoted to three-dimensional orthogonal drawings, little is known on the challenging task of extending to 3D the well-known topologyshape-metrics approach [18].

In order to reach such an ambitious research target, a crucial step is to find a three-dimensional counterpart of the well-known two-dimensional characterization of orthogonal representations (see, e.g. [18119]). More precisely, a solution to the following problem has to be found.

Let $G$ be a graph such that each edge is directed and labeled with a label in the set $\{U p$, Down, East, West, North, South $\}$. Does a three-dimensional orthogonal drawing of $G$ exist such that no two edges intersect and each edge has a direction consistent with its associated label?

This question has been addressed by Di Battista et al. 7/89 for simple classes of graphs, namely paths and cycles. The goal of this note is to shed some more light on the above basic question. Namely, we show that the known

\footnotetext{
* Work partially supported by European Commission - Fet Open project COSIN IST2001-33555 and by "Progetto Giovani Ricercatori: Algoritmi per la Visualizzazione di Grafi di Grandi Dimensioni" of the University of Perugia, Italy.
} 
characterization for cycles does not immediately extend to even seemingly simple graphs such as theta graphs, that is, graphs composed by two nodes of degree three and three disjoint paths, of length at least two, joining them. Also, we give a sufficient condition for recognizing three-dimensional orthogonal representations of theta graphs.

We remark that theta graphs have been studied extensively in the literature. For example, they arise in problems concerning graph planarity (see, e.g., [1,4 17] ), graph bandwidth (see, e.g., [12,14 16]), and chromatic polynomials (see, e.g., [2]).

\section{Preliminaries}

We assume familiarity with basic graph drawing terminology (see, e.g. 6]). A direction label is a label in the set $\{U, D, E, W, N, S\}$ specifying the directions Up, Down, East, West, North, South, respectively. Three distinct labels are also used to identify an octant of the reference system, which is assumed to be open.

Let $e$ be an undirected edge of a graph whose end-vertices are $u$ and $v$. We use the term darts for the two possible orientations $(u, v)$ and $(v, u)$ of edge $e$. A $3 D$ shape graph $\gamma$ is an undirected labeled graph such that (i) each dart is associated with one direction label; (ii) the two darts of the same edge have opposite labels; and (iii) each label in the set $\{U, D, E, W, N, S\}$ appears at least once in $\gamma$.

A three dimensional orthogonal drawing of a graph is such that vertices are mapped to grid points of an integer three dimensional grid and edges are segments along the integer grid lines connecting the end points. An intersection in a three dimensional orthogonal drawing is a pair of edges that overlap in at least a point that does not correspond to a common end-vertex.

A 3D shape graph $\gamma$ is simple if there exists an orthogonal drawing $\Gamma$ of $\gamma$ such that no two edges of $\Gamma$ intersect and each oriented edge satisfies the direction constraint defined by the direction labels associated with its darts. The simplicity testing problem for a 3D shape graph $\gamma$ is to decide whether $\gamma$ is simple.

Obviously, if $\gamma$ is simple, no vertex of $\gamma$ has two entering (or leaving) darts with the same label. For example, this implies that no vertex of $\gamma$ can have degree greater than six. Also, we restrict our attention to the class of shape graphs that do not have vertices of degree two whose entering darts are given opposite direction labels, since it can be shown (we omit the details for the sake of brevity) that each instance for which this property does not hold has an equivalent instance in this class.

Let $\gamma$ be a 3D shape graph. A flat $F \subset \gamma$ is a connected subgraph of $\gamma$ that is maximal with respect to the property that in every orthogonal drawing with the shape of $\gamma$ all edges in $F$ are co-planar. Observe that the labels of $\gamma$ come from the union of two oppositely directed pairs of directions. Also, the definition above extends the analogous definitions given in [789]. 
Let $\pi$ be a $3 \mathrm{D}$ shape path with vertices $p_{1}, p_{2}, \ldots, p_{k}$ and suppose to walk along $\pi$ from $p_{1}$ to $p_{k}$. The label path of $\pi$, denoted by $\sigma_{\pi}$, is the sequence of labels associated with darts $\left(p_{1}, p_{2}\right), \ldots,\left(p_{i}, p_{i+1}\right), \ldots,\left(p_{k-1}, p_{k}\right)$. Similarly, let $C$ be a $3 \mathrm{D}$ shape cycle with vertices $p_{1}, p_{2}, \ldots, p_{k}$ and suppose to walk along $C$ from $p_{1}$ to $p_{k}$. The label cycle of $C$, denoted by $\sigma_{C}$, is the sequence of label associated with darts $\left(p_{1}, p_{2}\right), \ldots,\left(p_{i}, p_{i+1}\right), \ldots,\left(p_{k-1}, p_{k}\right),\left(p_{k}, p_{1}\right)$.

Given a label path or cycle $\sigma$, a not necessarily consecutive subsequence $\tau \subseteq \sigma$, where $\tau$ consists of $k$ elements, is a canonical sequence 98 provided that: (i) $1 \leq k \leq 6$; (ii) the labels of $\tau$ are distinct; (iii) no flat of $\sigma$ contains more than three labels of $\tau$; and (iv) if a flat $F$ of $\sigma$ contains one or more labels of $\tau$, then $\tau \cap F$ form a consecutive subsequence of $\sigma$.

The following basic result for paths is given in [9]:

Theorem 1. 9] Let $\pi$ be a $3 D$ shape path with vertices $p_{1}, p_{2}, \ldots, p_{k}$ and let $\sigma_{\pi}$ be the label path associated with the sequence of darts $\left(p_{1}, p_{2}\right), \ldots,\left(p_{i}, p_{i+1}\right)$, $\ldots,\left(p_{k-1}, p_{k}\right)$. Shape path $\pi$ admits an intersection free orthogonal drawing with $p_{1}$ at the origin and $p_{k}$ in the UNE octant if and only if $\sigma_{\pi}$ contains a canonical sequence of three labels in the set $\{U, N, E\}$.

A characterization of simple shape cycles is given in $[8]$ :

Theorem 2. 8] A 3D shape cycle $C$ described by a sequence of direction labels $\sigma_{C}$ is simple if and only if it contains a canonical sequence of length six.

A theta graph is a graph with two non adjacent nodes of degree three and all other nodes of degree two [13]. Thus, a theta graph consists of two nodes of degree three and three disjoint paths, of length at least two, joining them. We will call $3 D$ theta shape a $3 \mathrm{D}$ shape for a theta graph.

In the following we will denote by $p$ and $q$ the two degree-three nodes of a theta shape. Also, given a shape path $\pi_{x}$ from $p$ to $q$, and a shape path $\pi_{y}$ from $q$ to $p$, we denote $C_{x, y}$ the shape cycle obtained by joining $\pi_{x}$ and $\pi_{y}$. Observe that given a theta shape, three paths $\pi_{x}, \pi_{y}$, and $\pi_{z}$, and three cycles $C_{x, y}, C_{x, z}$, and $C_{y, z}$, are defined.

\section{A Forbidden Theta Shape}

In this section we show that the simplicity of the cycles composing a theta shape does not imply the simplicity of the theta shape itself. We use the following notation: given a shape path $\pi$ (say $D W U N E)$ we will denote by $\bar{\pi}(\overline{D W U N E}$ in the example) the shape path obtained by orienting each edge in the opposite direction and changing its label with the opposite one $(\overline{D W U N E}=W S D E U)$. Also, we will use a dot to denote a series composition of paths (for example $\pi_{1}=\pi_{2} \cdot \bar{\pi}_{3}$ ). Given two distinct vertices $v$ and $w$ of a shape path (shape cycle, theta shape, respectively) $\gamma$, we say that $v$ is $Y$ with respect to $w$, where $Y \in\{U, D, E, W, N, S\}$, if in any drawing of $\gamma$, denoted $\Pi_{v}$ and $\Pi_{w}$ the two planes orthogonal to $Y$ containing $v$ and $w$ respectively, the two planes may be joined by a segment oriented from $\Pi_{w}$ to $\Pi_{v}$ which has direction $Y$. Observe that, if $v$ is $Y$ with respect to $w, w$ is $\bar{Y}$ with respect to $v$. 


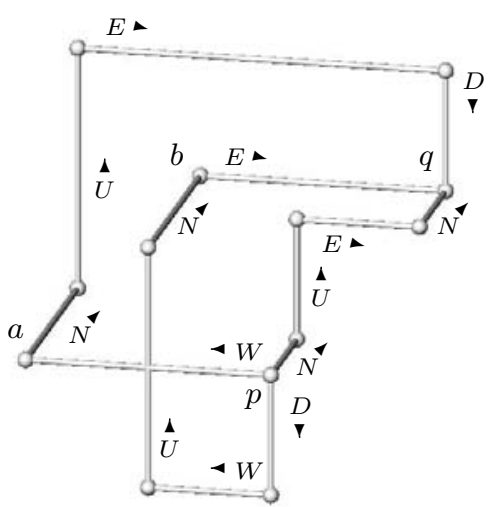

(a)

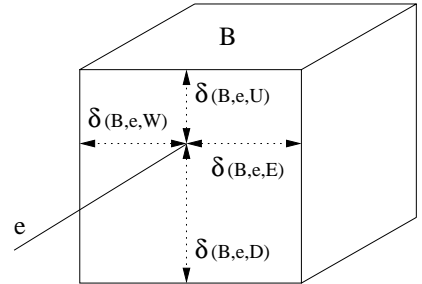

(b)

Fig. 1. (a) A not simple theta shape and (b) the definition of $\delta(B, e, X)$.

Theorem 3. There exists a 3D shape graph that is not simple even if all its induced cycles are simple.

Proof. Let $\vartheta$ be the theta shape composed by the three paths: $\pi_{1}=W N U E D$, $\pi_{2}=D W U N E$, and $\pi_{3}=N U E N$ (see Fig. 1 a). The three cycles $C_{1,2}, C_{1,3}$, and $C_{2,3}$ defined by $\vartheta$ are simple. Namely, we have:

$C_{1,2}=W N U E D \cdot \overline{D W U N E}=W N U E D W S D E U$

$C_{1,3}=W N U E D \cdot \overline{N U E N}=W N U E D S W D S$

$C_{2,3}=D W U N E \cdot \overline{N U E N}=D W U N E S W D S$

and each of them satisfies the hypotheses of Theorem 2. In fact, $C_{1,2}$ contains, for example, the canonical sequence identified by the checked labels in the sequence $W \check{N} U E \check{D} \check{W} \check{S} D \check{E} \check{U} ; C_{1,3}$ contains, for example, the canonical sequence identified by the checked labels in the sequence $W \check{N} \check{U} \check{E} D \check{S} \breve{W} \check{D} S$; and $C_{2,3}$ contains, for example, the canonical sequence identified by the checked labels in the sequence $D \check{W} \check{U} \check{N} \check{E} S W \check{D} \check{S}$.

Suppose for a contradiction that $\vartheta$ is simple. Consider a non intersecting orthogonal drawing $\Gamma$ of $\vartheta$. Let $\sigma$ be the label path $E N U E N W$ from $a$ to $b$. By Theorem 1, since the only canonical sequences of length three contained in $\sigma$ are of type NUW or NUE, then $b$ is NUW or NUE with respect to $a$ in each non intersecting drawing of $\sigma$. Let $\sigma^{\prime}$ be the label path $N U E D W$ from $a$ to $b$. By Theorem [, since the only canonical sequences of length three contained in $\sigma^{\prime}$ are of type NUE, NDE, or NDW, then $b$ is NUE, NDE, or NDW with respect to $a$ in each non intersecting drawing of $\sigma^{\prime}$. Let $\sigma^{\prime \prime}$ be the label path $E D W U N$ from $a$ to $b$. By Theorem 1, since the only canonical sequences of length three contained in $\sigma^{\prime \prime}$ are of type NUW, NDE, or NDW, then $b$ is NUW, $\mathrm{NDE}$, or NDW with respect to $a$ in each non intersecting drawing of $\sigma^{\prime \prime}$. We have a contradiction, the three conditions above can not be simultaneously verified and thus one intersection necessarily occurs. 


\section{Triply Expanding Drawings}

Let $\pi_{1}, \pi_{2}, \ldots, \pi_{n}$ be $n$ shape paths starting from a common point $p$. Denote by $m_{i}$ the number of edges of $\pi_{i}$, and by $e_{i, h}$, with $h=1, \ldots, m_{i}$, the $h$-th edge of $\pi_{i}$ starting from $p$. An expanding drawing of $\pi_{1}, \pi_{2}, \ldots, \pi_{n}$ is a simple 3D orthogonal drawing for which edges $e_{1, m_{1}}, e_{2, m_{2}}, \ldots, e_{n, m_{n}}$ can be replaced by arbitrarily long segments without creating any intersection with the drawing. The bounding box of an expanding drawing is the bounding box of the drawing when edges $e_{1, m_{1}}, e_{2, m_{2}}, \ldots, e_{n, m_{n}}$ are removed. In [8] Di Battista et al. showed the following sufficient condition for the existence of an expanding drawing of two paths (doubly expanding drawing).

Theorem 4. 8] A shape path $\pi$ with $n$ edges admits a doubly expanding drawing if it either consists of exactly two edges or contains at least two flats.

A triply expanding drawing is an expanding drawing of three paths. The next theorem extends Theorem 4 to triply expanding drawings.

Theorem 5. Let $\pi_{x}, \pi_{y}$, and $\pi_{z}$ be three shape paths starting from a common point $p$. If the paths $\overline{\pi_{x}} \cdot \pi_{y}, \overline{\pi_{x}} \cdot \pi_{z}$, and $\overline{\pi_{y}} \cdot \pi_{z}$ consist of exactly two edges or contain at least two flats, then $\pi_{x}, \pi_{y}$, and $\pi_{z}$ admit a triply expanding drawing.

Due to space limitations we omit the proof of Theorem 5 and refer to [10] the interested reader. Roughly, the theorem can be proved by construction, after a suitable case analysis, and by using the following lemma.

Lemma 1. A shape graph $\gamma$ that admits a three dimensional orthogonal drawing such that no intersection occurs between two edges of the same flat is simple.

Proof. The statement can be proved by construction. Namely, given a three dimensional orthogonal drawing $\Gamma$ such that no intersection occurs between two edges of the same flat, an iterative process can be used to produce an orthogonal drawing $\Gamma^{\prime}$ without intersections. Consider an intersection between two edges $e_{1}$ and $e_{2}$ that do not belong to the same flat. The following technique eliminates the intersection, without introducing a new one. Consider a plane $p$ common to $e_{1}$ and $e_{2}$ and a direction $d$ orthogonal to $p$. Move one unit in the $d$ direction all the nodes in the open half-space determined by $p$ and $d$, the end-points of $e_{1}$, and the end-points of all the edges of the flat $F$ of $e_{1}$ perpendicular to $d$, if any. It can be shown that the obtained drawing is a $3 \mathrm{D}$ orthogonal drawing of $\gamma$, that the intersection between $e_{1}$ and $e_{2}$ has been removed, and that no other intersection has been introduced (see [10] for details).

By virtue of the preceding lemma, intersections occurring between edges (and nodes) that do not share a flat can be neglected, assuming that they could be easily eliminated in a post processing step. 


\section{A Sufficient Condition for Theta Shapes Simplicity}

Let $\Theta$ be a theta shape composed by three shape paths $\pi_{1}, \pi_{2}$, and $\pi_{3}$ from point $p$ to point $q$. Let $e_{i j}$, with $j=1, \ldots, m_{i}$, the $j$-th edge of $\pi_{i}$. In the following we denote by $l_{i j}$ the label associated with $e_{i j}$ when directed according to $\pi_{i}$. Recall that when $\pi_{i}$ is reversed, as in $C_{k, i}=\pi_{k} \cdot \overline{\pi_{i}}$, the label associated with $e_{i j}$ is the opposite of $l_{i j}$, that is $\overline{l_{i j}}$.

Theorem 6. Let $\Theta$ be a theta shape composed by three shape paths $\pi_{1}, \pi_{2}$, and $\pi_{3}$ from point $p$ to point $q$. If for each $\pi_{i}$ there exist three edges $e_{i 1}, e_{i 2}$, and $e_{i 3}$, such that for each pair of paths $\pi_{i}$ and $\pi_{j}, i, j=1,2,3, i \neq j$, the six labels $l_{i 1}, l_{i 2}, l_{i 3}, \overline{l_{j 1}}, \overline{l_{j 2}}$, and $\overline{l_{j 3}}$ form a canonical sequence $\tau_{i, j}$ for the shape cycle $C_{i, j}=\pi_{i} \cdot \overline{\pi_{j}}$, then $\Theta$ is simple.

Proof. By using the canonicity of $\tau_{1,2}, \tau_{1,3}$, and $\tau_{2,3}$, the following properties for labels $l_{i j}$ can be verified:

1. The labels $l_{i j}$ of the same path are different, i.e., $l_{i j} \neq l_{i k}, i, j, k=1,2,3$, $j \neq k$.

2. No two labels $l_{i j}$ of the same path are opposite, i.e., $l_{i j} \neq \overline{l_{i k}}, i, j, k=1,2,3$, $j \neq k$.

3. No two labels $l_{i j}$ of different paths are opposite, i.e., $l_{i j} \neq \overline{l_{h k}}, i, j, h, k=$ $1,2,3, i \neq h$.

It follows that, for each $i=1,2,3$, labels $l_{i 1} l_{i 2} l_{i 3}$ are a permutation of the same three labels, one for each oppositely directed pair. We assume, without loss of generality, that $l_{i j} \in\{U, N, E\}$ with $i, j=1,2,3$.

The nine edges $e_{i j}(i, j=1,2,3)$ bound eight connected subgraphs of the theta graph. We call $G_{i, j}$ the subpath of $\pi_{i}$ from $e_{i j}$ to $e_{i(j+1)},(i=1,2,3$, $j=1,2)$. Further, we call $G_{p}$ the subgraph composed by the three paths from $p$ to $e_{i 1}$ with $i=1,2,3$, and $G_{q}$ the subgraph composed by the three paths from $e_{i 3}$ with $i=1,2,3$ to $q$.

Observe that $G_{i, j}$, with $i=1,2,3$, and $j=1,2$ admits a doubly expanding drawing. In fact, since the labels associated with $e_{i j}$ and $e_{i(j+1)}$ are canonical, it follows that, if they are not consecutive, they do not share a flat, and Theorem 4 applies. Also, observe that $G_{p}$ admits a triply expanding drawing. In fact, since, for each pair $i, j, e_{i 1}$ and $e_{j 1}$ are canonical in $C_{i, j}$, it follows that, if they are not consecutive, they do not share a flat, and Theorem 5 applies. Analogously, $G_{q}$ admits a triply expanding drawing.

We draw a doubly expanding drawing $\Gamma_{i, j}$ for each $G_{i, j}$, with $i=1,2,3$, and $j=1,2$. Theorem 4 Let $L$ be the maximum length of a side of a bounding box of the drawings obtained above. We draw a triply expanding drawing $\Gamma_{p}$ of $G_{p}$ and a triply expanding drawing $\Gamma_{q}$ of $G_{q}$.

Now we show how to set the lengths $\lambda_{i j}, i, j=1,2,3$, of the part of each edge $e_{i j}$ that is not contained in the bounding box of any expanding drawing, since the actual lengths of the edges can be easily computed from it.

Denote by $B_{p}, B_{q}$, and $B_{i, j}$, with $i=1,2,3$ and $j=1,2$, the bounding boxes of $\Gamma_{p}, \Gamma_{q}$, and $\Gamma_{i, j}$, respectively. Since edges $e_{i 1}$, with $i=1,2,3$, have labels in 
$\{U, N, E\}$, there are three pairwise orthogonal sides of $B_{p}$ from which edges $e_{i 1}$ may come out. Denote by $v_{B_{p}}$ the only point common to these sides. Denote by $v_{B_{q}}$ the point common to the analogous sides of $B_{q}$. Place the drawings $\Gamma_{p}$ and $\Gamma_{q}$ in such a way that $v_{B_{p}}$ has coordinates $(-2 L-1,-2 L-1,-2 L-1)$ and $v_{B_{q}}$ has coordinates $(2 L+1,2 L+1,2 L+1)$.

Now we show how to add the drawing of $\Gamma_{i, 1}$ and $\Gamma_{i, 2}$, for $i=1,2,3$. Given a bounding box $B$, an edge $e$ protruding from it, and a direction label $X$ orthogonal to $e$, we denote by $\delta(B, e, X)$ the distance of $e$ from the side of $B$ in the $X$ direction (see Figure 1,b). We determine $\lambda_{i j}$, with $i, j=1,2,3$, by considering the following equalities.

$$
\begin{aligned}
\lambda_{i, 1}= & 4 L+2+\delta\left(B_{q}, e_{i 3}, \overline{l_{i 1}}\right)-\delta\left(B_{i, 2}, e_{i 3}, \overline{l_{i 1}}\right)+ \\
& +\delta\left(B_{i, 2}, e_{i 2}, \overline{l_{i 1}}\right)-\delta\left(B_{i, 1}, e_{i 2}, \overline{l_{i 1}}\right) \\
\lambda_{i, 2}= & 4 L+2+\delta\left(B_{p}, e_{i 1}, l_{i 2}\right)+\delta\left(B_{q}, e_{i 3}, \overline{l_{i 2}}\right)- \\
& -\delta\left(B_{i, 1}, e_{i 1}, l_{i 2}\right)-\delta\left(B_{i, 2}, e_{i 3}, \overline{l_{i 2}}\right) \\
\lambda_{i, 3}= & 4 L+2+\delta\left(B_{p}, e_{i 1}, l_{i 3}\right)-\delta\left(B_{i, 1}, e_{i 1}, l_{i 3}\right)+ \\
& +\delta\left(B_{i, 1}, e_{i 2}, l_{i 3}\right)-\delta\left(B_{i, 2}, e_{i 2}, l_{i 3}\right)
\end{aligned}
$$

Now we show that no intersection between edges sharing a flat has been introduced in the drawing. By Theorems 4 and 5 no intersection occurs in each (doubly or triply) expanding drawing.

There is no intersection between two edges of the same $\pi_{i}$. In fact, since $0 \leq \delta\left(B_{i, j}, e, X\right) \leq L$, and by equations 1,2 , and 3 , each $\lambda_{i j}$, with $i, j=1,2,3$ is at least $2 L+2$ and each one of $B_{p}, B_{q}, B_{i, 1}$, and $B_{i, 2}$ is in a different octant.

Intersections between edges belonging to different paths involve edges that do not share a flat, and thus, by Lemma 1, can be removed. Indeed, suppose for a contradiction that $e_{x}$ and $e_{y}$ are two intersecting edges belonging to two different paths $\pi_{x}$ and $\pi_{y}$ and that $e_{x}$ and $e_{y}$ are on the same flat $F$. Edges $e_{x}$ and $e_{y}$ do not belong to the same expanding drawing, since otherwise they could not intersect.

There must exist a path $\pi^{*}$ joining $e_{x}$ and $e_{y}$, entirely contained in $F$, and containing $p$ or $q$. In $\pi^{*}$ there can be at most three of the edges $e_{i j}$, with $i=x, y$, and $j=1,2,3$, since otherwise there would be more than three canonical labels on the same flat for the cycle $C_{x, y}$, contradicting the hypothesis that $\tau_{x, y}$ is a canonical sequence. Since edges $e_{i j}$ in $\pi^{*}$ are on the same flat $F$ and are canonical, they are necessarily adjacent, and thus if they are consecutive they are orthogonal with each other. If $e_{x}$ and $e_{y}$ coincide with two edges $e_{i j}$, then they can not intersect since they are parallel or adjacent and orthogonal. Otherwise, since edges $e_{i j}$ are the only ones to transition between octants, then $e_{x}$ and $e_{y}$ belong to two different octants and they can not intersect. 


\section{References}

1. D. S. Archdeacon and J. Šráň. Characterizing planarity using theta graphs. $J$. Graph Theory, 27:17-20, 1998.

2. J. Brown, C. Hickman, A. Sokal, and D. Wagner. On the chromatic roots of generalized theta graphs. J. of Combinatorial Theory, Series B, to appear.

3. I. Bruß and A. Frick. Fast interactive 3-D graph visualization. In F. J. Brandenburg, editor, Graph Drawing (Proc. GD '95), volume 1027 of Lecture Notes Comput. Sci., pages 99-110. Springer-Verlag, 1996.

4. G. Chartrand and F. Harary. Planar permutation graphs. Ann. Inst. H. Poincaré, Sect B, 3:433-438, 1967.

5. I. F. Cruz and J. P. Twarog. 3D graph drawing with simulated annealing. In F. J. Brandenburg, editor, Graph Drawing (Proc. GD '95), volume 1027 of Lecture Notes Comput. Sci., pages 162-165. Springer-Verlag, 1996.

6. G. Di Battista, P. Eades, R. Tamassia, and I. G. Tollis. Graph Drawing. Prentice Hall, Upper Saddle River, NJ, 1999.

7. G. Di Battista, G. Liotta, A. Lubiw, and S. Whitesides. Embedding problems for paths with direction constrained edges. In Annual International Computing and Combinatorics Conference, (COCOON 2000), volume 1858 of Lecture Notes Comput. Sci., pages 64-73. Springer-Verlag, 2000.

8. G. Di Battista, G. Liotta, A. Lubiw, and S. Whitesides. Orthogonal drawings of cycles in 3d space. In J. Marks, editor, Graph Drawing (Proc. GD '00), volume 1984 of Lecture Notes Comput. Sci. Springer-Verlag, 2001.

9. G. Di Battista, G. Liotta, A. Lubiw, and S. Whitesides. Embedding problems for paths with direction constrained edges. J. of Theor. Comp. Sci., 2002. to appear.

10. E. Di Giacomo, G. Liotta, and M. Patrignani. On orthogonal 3d shapes of theta graphs. Tech. Report RT-DIA-71-2002, Dept. of Computer Sci., Univ. di Roma Tre, 2002. http://web.dia.uniroma3.it/research/.

11. D. Dodson. COMAIDE: Information visualization using cooperative 3D diagram layout. In F. J. Brandenburg, editor, Graph Drawing (Proc. GD '95), volume 1027 of Lecture Notes Comput. Sci., pages 190-201. Springer-Verlag, 1996.

12. D. Eichhorn, D. Mubayi, K. O'Bryant, and D. B. West. Edge-bandwidth of theta graphs. J. Graph Theory, 35:89-98, 2000.

13. F. Harary. Graph Theory. Addison-Wesley, Reading, Mass., 1969.

14. T. Jiang, D. Mubayi, A. Shastri, and D. B. West. Edge-bandwidth of graphs. SIAM Journal on Discrete Mathematics, 12(3):307-316, 1999.

15. A. Papakostas and I. G. Tollis. Algorithms for incremental orthogonal graph drawing in three dimensions. Journal of Graph Algorithms and Applications, 3(4):81115, 1999

16. G. Peck and A. Shastri. Bandwidth of theta graphs with short paths. Discrete Mathematics, 103, 1992.

17. I. Sciriha and S. Fiorini. On the characteristic polynomial of homeomorphic images of a graph. Discrete Mathematics, 174:293-308, 1997.

18. R. Tamassia. On embedding a graph in the grid with the minimum number of bends. SIAM J. Comput., 16(3):421-444, 1987.

19. G. Vijayan and A. Wigderson. Rectilinear graphs and their embeddings. SIAM J. Comput., 14:355-372, 1985. 